\title{
Application Advances of Contrast-enhanced Ultrasound in Clinical Diagnosis and Treatment
}

\author{
Zhou Xiaoyu ${ }^{1}$, Peng Changfu ${ }^{2, *}$ \\ ${ }^{1}$ Department of Medical College, Hunan Provincial People's Hospital, The First Affiliated Hospital of Hunan Normal University, Changsha, \\ China \\ ${ }^{2}$ Center for Ophthalmic Optics, Hunan Provincial People's Hospital, Changsha, China
}

Email address:

1341798294@qq.com (Zhou Xiaoyu),oculistpeng@yahoo.com (Peng Changfu)

${ }^{*}$ Corresponding author

\section{To cite this article:}

Zhou Xiaoyu, Peng Changfu. Application Advances of Contrast-enhanced Ultrasound in Clinical Diagnosis and Treatment. International Journal of Medical Imaging. Vol. 9, No. 2, 2021, pp. 98-103. doi: 10.11648/j.ijmi.20210902.11

Received: April 5, 2021; Accepted: April 15, 2021; Published: April 23, 2021

\begin{abstract}
Because the affected parts of some tumors and diseases are generally hidden, some are deeper, and the tissue structure is more complex and diverse, misdiagnosis and missed diagnosis are very easy to occur in the process of clinical diagnosis. In addition, the development of various diseases and tumors is closely related to the quality of life and prognosis of patients. Therefore, the diagnosis and treatment of diseases and monitoring after treatment are particularly important for patients. Ultrasound is easy to operate, non-invasive, and can be observed in real time. Ultrasound is the most used Frequent imaging diagnostic tools. In recent years, contrast-enhanced ultrasound (CEUS), as a newly developed imaging technology, has made important progress in ultrasound examination. In particular, the second-generation microbubble (MB) contrast agent in contrast-enhanced ultrasound (CEUS) has been widely used in clinical practice. It can observe the whole process of organ and tumor microcirculation blood perfusion in real time and continuously, not only The morphological characteristics of the lesion can also provide the blood flow characteristics of the lesion, which improves the ability of the ultrasonic diagnostic instrument to display the blood flow in the lesion. Due to its non-invasiveness, real-time and safety, contrast-enhanced ultrasound technology provides safe, effective and valuable diagnostic and treatment options for ophthalmology, liver, gastrointestinal tract, heart, pediatrics and other fields. This article briefly reviews the origin and development of contrast agent ultrasound, basic principles, safety, the application of contrast-enhanced ultrasound in clinical diagnosis and treatment, and the advantages and disadvantages of contrast-enhanced ultrasound, and discusses its future development and limitations for pre-clinical surgery The qualitative diagnosis, selection of surgical methods and prognostic judgment and evaluation provide more accurate and comprehensive information in order to better serve and treat clinical diagnosis.
\end{abstract}

Keywords: Contrast-enhanced Ultrasound, Ultrasound Contrast Agent, Clinical Application

\section{Introduction}

The discovery of contrast-enhanced ultrasound (CEUS) can be traced back to 1968. After Gramiak and Shah injected normal saline through the intra-aortic catheter, a "bubble cloud" appeared in the aortic root. Therefore, the physical properties of UCA were discovered. [1] Early researchers, including Bove and Kremkau, later laid the foundation for the development of ultrasound contrast agents (UCA) and led to the identification of these agents as true, non-diffusible intravascular indicators. Once the physical properties of UCA were established, investigators looked at its utility. DeMaria et al. was the first to apply CEUS to the heart field. In the end, the discovery and practicability of the first generation of UCA led to the rapid development of CEUS. Sidhu PS et al. published the 2017 updated version of the European Federation of Medical and Biological Ultrasound Societies (EFSUMB) practice guidelines for the use of CEUS in non-liver applications. In these guidelines, EFSUMB proposed to use CEUS for pancreatic masses, endoscopic ultrasound examination, gastrointestinal tract, spleen, genitourinary system assessment, etc., and then continue to improve and promote the development of CEUS [2]. 


\section{Principles of Contrast Ultrasound}

In an ultrasound imaging system, microbubbles act as resonant scatterers, increasing the backscattered signal by up to $30 \mu \mathrm{dB}$, and generating echoes with characteristic harmonics, causing dramatic changes in the reflection mode of acoustic impedance in the tissue or blood. UCA produces a significant signal-to-noise ratio and enhances the ultrasound signal, thereby greatly improves the quality. Due to the driving amplitude of the ultrasonic pulse, the microbubble exhibits a unique echo reflection mode, which mainly includes the nonlinear fundamental wave, high-order harmonics, sub-harmonics and second harmonics. At present, the second harmonic is the most commonly used in clinical practice. Harmonic imaging [3]. The second harmonic is usually used to enhance the diagnostic image, because the microbubble scattering is nonlinear at all amplitudes, and its adjacent tissue structures will not resonate in the form of microbubbles. The adjacent normal tissue ultrasound is at relatively low amplitude (lower amplitude). MI) shows a linear response at frequency. Therefore, ultrasound manufacturers use the nonlinear response of microbubbles to design new ultrasound systems that can provide ultrasound pulse sequences of different amplitudes, phases, and frequencies to reflect the non-linearity of ultrasound contrast agents. Linear response and linear response of the organization. Using the best low mechanical index (MI) setting can minimize the damage of microbubbles. Once the contrast agent shell ruptures and the gas diffuses, the microbubbles lose their scattering properties and are no longer an effective contrast agent. The destruction of microbubbles will result in a decrease in time- and intensity-related contrast, which will not only reduce image quality, but also shorten the duration of contrast. Then a specific algorithm can be used to process the received data and draw an image that shows the flow pattern of microbubbles, which corresponds to the blood flow in the target organ or tissue, thereby reducing the clutter from scattered ultrasound and enhancing the blood vessels contrast. The interaction between tissues effectively enhances the imaging effect of microvessels, thereby improving the accuracy of ultrasound diagnosis [4].

\section{Ultrasound Contrast Agent}

Ultrasound contrast agent (UCA) is composed of microbubbles encased in an inert and relatively insoluble gas. The exterior is coated with a lipid shell. The enclosed gas is usually perfluorinated (octafluoropropane) or sulfur hexafluoride, which is characterized by a high density, high molecular weight gas that exhibits low solubility. The protein or lipid shell provides enhanced stability, thereby improving durability and functionality. The shell reduces the dissolution or destruction of microbubbles in the blood. When hit by ultrasound, the microbubbles will oscillate and generate an acoustic signal, which will be detected and reproduced on the ultrasound image [5]. The diameter of microbubbles usually ranges from 1 to $10 \mu \mathrm{m}$ (red blood cells are about $7.8 \mu \mathrm{m}$ in diameter), thus allowing unobstructed passage through the pulmonary blood vessels from the peripheral injection site, and then into the left ventricle and into the systemic circulation [6]. At the same time, due to their micron diameter and the significant difference in the acoustic impedance at the blood-gas interface, they scatter the ultrasonic beam very strongly. The size of a single UCA is not allowed to pass through the intravascular boundary, so it is still a true intravascular indicator. Based on its ability to pass through the lungs and long half-life in the human body, three generations of ultrasound contrast agents have been developed. The first generation of agents are air-filled microbubbles, such as saline, indocyanine green, hydrogen peroxide, and dextran ultrasonic solution compounds [7]. These microbubbles are usually too large to pass through the pulmonary circulation or very unstable. They usually require high sound power to oscillate or destroy their microbubbles and cannot be used clinically. Later, Feinstein et al [8]. found microbubbles that were small and stable enough to pass through the pulmonary circulation and reach the left ventricle (LV). This enabled the first commercial UCA to come out in the 1980s, including Levovist, whose gas composition is mainly nitrogen, which is relatively diffusible. The second-generation UCA produced afterwards includes Optison (1997), Definity (2001), Sonovue (2001), Lumason (2014). Optison is composed of type A protein microspheres and is usually administered by large-dose injection through a peripheral vein. Sonovue and Lumason lipid shells encapsulate sulfur hexafluoride gas. Sonazoid also has perfluorobutane gas encapsulated in the same lipid shell microspheres. The second-generation reagents are composed of inert gases with lower solubility, so they last longer in the cycle. The shell of these contrast agents will be destroyed by lower MI oscillations to release microbubbles, so they are suitable for ultrasound contrast. The third-generation agent, such as EchogenTM, encapsulates genes, drugs and other substances in a lipid shell. Once it reaches the temperature of the human body, it can be transformed from a liquid form to a gas form, which has a targeted therapeutic effect. However, these reagents are not widely used as they are in the research and development stage.

\section{The Safety of CEUS}

As mentioned earlier, UCA is safe and has a very low incidence of side effects. Since there is no cardiac, liver or nephrotoxic effects, laboratory tests are not required to assess liver, kidney or thyroid function before administration. The incidence of serious adverse events is lower than current $\mathrm{X}$-ray contrast agents. According to reports, the incidence of life-threatening allergic reactions in abdominal applications is $0.001 \%$, and further studies have reproduced this very low incidence of adverse events. Wei et al. also noted that the incidence of serious adverse events (SAE) in patients receiving UCA was extremely low, only $0.01 \%$ [9]. In June 2008, the FDA and the manufacturers of Optison and 
Definity jointly released three separate safety studies to further assess the risks of each UCA. These studies included a retrospective observational study that showed that there was no significant increase in mortality with uca for contrast-enhanced ultrasound, no change in pulmonary artery pressure, and no SAE [6]. Wever-Pinzon et al. reviewed 1513 consecutive hospitalized patients with pulmonary hypertension who received UCA. Only three patients had SAEs after taking UCA, and none of these events were directly attributable to UCA itself [10]. Research on the safety of SonoVue ultrasound contrast agent shows that the use of large doses of SonoVue contrast agent will not aggravate the attenuation of his heart and lung function [11]. When performing ultrasound contrast, emergency rescue tools and facilities should be available to deal with the possibility of adverse events in patients, and contrast operators should be trained in resuscitation, so that if serious adverse reactions occur, such as anaphylactic shock, they are capable.

\section{Clinical Applications of Contrast-enhanced Ultrasound in Ophthalmology}

Fundus diseases such as choroidal hemangioma and choroidal melanoma are easily confused in clinical diagnosis [12]. Contrast-enhanced ultrasound can effectively diagnose and differentiate choroidal tumors. Guo Peiqi's research found that choroidal hemangioma has the highest echo, which is uniformly hyperechoic, and the concentration of contrast agent in the lesion is higher than that of choroidal melanoma. Ultrasound is a powerful non-invasive tool for evaluating vitreoretinal diseases and diagnosing intraocular tumors. A blood clot in the vitreous can cause part of the retinal detachment. It is difficult to distinguish between retinal detachment and vitreous membrane. Han reported that only B-mode ultrasound had a diagnostic accuracy of $78 \%$ and color Doppler was $81 \%$. The use of the first generation of ultrasound contrast agents significantly improved the ultrasound recognition of retinal detachment, increasing its sensitivity from $57 \%$ to $93 \%$ [13]. Bertolotto $M$ et al. found that the use of CEUS and second-generation contrast agents had identified retinal detachment with $100 \%$ sensitivity. And it was also found that in the case of severe vitreous hemorrhage with conventional gray-scale ultrasound and Doppler mode, or the opacity of the medium, the thrombosis of tumor blood vessels and the lack of blood vessels or partial necrosis of the disease, a more accurate judgment cannot be made [14]. CEUS effectively assesses the vascularization of choroidal melanomas to distinguish them from choroidal nevi. CEUS can also effectively monitor the response of radiotherapy to melanomas, showing that the blood supply to the lesions is gradually decreasing. Yang Wenli et al. found that the sensitivity of contrast-enhanced ultrasound for choroidal melanoma and choroidal hemangioma was 90.5\% (19/21), and the specificity was $12 / 14$ [15]. Studies have shown that when performing a contrast-enhanced ultrasound examination of the eye, one should be extra careful to avoid the risk of non-thermal effects [16]. Generally speaking, microbubble contrast agents are very safe. They have few side effects and no interaction with the thyroid. The incidence of severe hypersensitivity or allergic reactions is lower than that of current MR contrast agents. Theoretically, the interaction between the ultrasound beam and the ultrasound contrast agent may produce biological effects. Data from small animal models indicate that microvessel rupture may occur when sonar microbubbles are under high MI [17]. Therefore, EFSUMB's guidelines for the use of CEUS recommend that careful use in the eyes to avoid damage to the vasculature.

\section{The Clinical Application of Contrast-enhanced Ultrasound in Other Disciplines}

\subsection{Diagnosis of Gastrointestinal Diseases}

The popularity and development of contrast-enhanced ultrasound (CEUS) makes contrast-enhanced ultrasound an excellent choice for routine and emergency imaging examinations for gastrointestinal diseases. In terms of estimating the disease activity of Crohn's disease, CEUS can evaluate and quantify the enhancement of Crohn's disease in different parietal layers, and is related to the clinical activity index (CDAI), with good sensitivity and sensitivity [18]. Carla Serra [19] found that CEUS had $81.0 \%$ sensitivity in evaluating Crohn's Disease Activity Index (CDAI), with specificities of $63.0 \%$ and $55.6 \%$, respectively. The positive predictive values of patients with active and inactive diseases were $63.0 \%$ and $58.6 \%$, and the negative predictive values were $81.0 \%$ and $78.9 \%$. CEUS assessment of intestinal wall vascular disease may be a sensitive tool to detect inflammatory activity. Migaleddu et al. found that CEUS is evaluating the inflammatory activity of $47 \mathrm{CD}$ patients, and found that CEUS has high sensitivity and specificity in detecting inflammatory activity based on the CDAI score. In addition, CEUS can also distinguish fibrosis and inflammatory stenosis, characterize complications such as abscesses and fistulas, and monitor treatment [20].

\subsection{Application of CEUS in Liver Tumors}

Contrast-enhanced ultrasound can be used in the differential diagnosis of liver tumors, and is very helpful in clinical practice that lacks B-line scan or power Doppler morphological standards. Strobel [21] found that contrast-enhanced ultrasound can correctly assess 723/755 malignant lesions (sensitivity of $95.8 \%$ ) and $476 / 573$ benign lesions (specificity of $83.1 \%$ ). At the same time, it has been reported in the literature that the positive predictive value of CEUS for focal liver disease (FLL) $\leq 20 \mathrm{~mm}$ is $84.9 \%$. 
CEUS has high diagnostic accuracy in the differential diagnosis of small and subgravity FLL in clinical practice [22]. According to reports, CEUS-guided biopsy can avoid sampling of necrotic materials by guiding the biopsy to the contrast-enhanced tissue inside the tumor, or by identifying previously undiagnosed tissues to increase the diagnostic accuracy by up to $10 \%$. Visualized lesions are easier to biopsy. If a residual tumor is found, the needle can be repositioned to the residual tumor under the guidance of CEUS before ablation. In follow-up studies, CEUS can be used to identify a small amount of residual tumors immediately after treatment. CEUS is also used for percutaneous hepatic cholangiography and drainage (PTCD) for hepatobiliary examinations such as biliary imaging and fistula angiography [23].

\subsection{Application of Contrast-enhanced Ultrasound in Cardiovascular Diseases}

The American Society of Echocardiography (ASE) issued practical guidelines for the application of CEUS in 2001. These guidelines were updated in 2014, recommending the use of CEUS to improve the visibility of the endocardium. Contrast-enhanced ultrasound (CEUS) is increasingly used to assess the differential diagnosis of known or suspected arteriosclerosis and cardiac tumors and thrombosis, as well as to assess valve function. Myocardial contrast-enhanced ultrasound improves the temporal and spatial resolution of myocardium, and is also effective in diagnosing myocardial infarction, distinguishing heart tumors and thrombus [24]. In terms of vascular imaging, contrast-enhanced ultrasound is easy to operate, can accurately and effectively detect plaque vulnerability, carotid atherosclerosis, and the degree of new blood vessel formation and stenosis in plaque, and can predict cardiovascular and cerebrovascular diseases. In patients with myocardial infarction, CEUS can be used to assess thrombosis in the heart, which may have important clinical significance. For patients with known or suspected carotid atherosclerosis, CEUS can quantitatively evaluate new blood vessels and plaque ulcers in carotid atherosclerotic plaques, and identify high-risk plaques that cause clinical events [25].

\subsection{Application of Contrast-enhanced Ultrasound in Pediatrics}

The use of contrast-enhanced ultrasound (CEUS) in children is supported by clinical experience and evidence in the field of pediatrics. More and more evidence shows that CEUS is safe for children [26]. The European Federation of Ultrasound and Medicine (EFSUMB) has evaluated the current status of CEUS in children, discussed the current status and development of CEUS and made recommendations. The US Food and Drug Administration (FDA) has approved Lumason ${ }^{\mathrm{TM}}$ for pediatric liver imaging, which is an important development in pediatric imaging and can significantly reduce ionizing radiation exposure in many areas [27]. CEUS can now also be used to assess focal liver disease (FLL) in children. Similar to adults, the pediatric diagnostic practice of CEUS may have the same accuracy as contrast CT and CT in FLL assessment. You can also perform targeted biopsy and accurately sample tissues. CEUS can enhance the discrimination ability of children with cavitary pneumonia through color and power Doppler imaging [28]. CEUS has a higher sensitivity in the diagnosis of splenic laceration, rupture, fracture or active bleeding in the evaluation of splenic parenchyma in children's vein and delayed phase [29]. The unique and well-known advantages of contrast-enhanced ultrasound will promote the use of CEUS in children's imaging in the future.

\section{Advantages and Disadvantages of Contrast-enhanced Ultrasound}

Compared with other imaging modes (such as CECT and CEMRI), contrast-enhanced ultrasound has the advantage of strong real-time imaging capabilities under the condition of full control of the operation, avoiding the arterial phase errors sometimes observed on CECT and CEMRI. Ultrasound imaging is relatively cost-effective. The advantages of low temperature, wide application range, portability, fixed or bedside use, no ionizing radiation, and high patient acceptance (especially pediatric patients who do not require sedation and/or intubation) are also suitable for EUS. Since microbubbles are not cleared or excreted in the kidneys, they can be used in patients with impaired renal function. Similarly, they do not induce thyroid storms like iodo-based DCT contrast agents, because they do not interact with thyroid metabolism. However, CEUS is limited in evaluating lesions smaller than $10 \mathrm{~mm}$, deep $(>8 \mathrm{~cm})$ or subdiaphragmatic lesions, and general patients. Patients who are uncooperative (including patients with dyspnea) or patients with interfering intestinal gas have one injection Usually only one lesion can fully show the characteristics of all vascular stages, and then additional contrast agent injection is required to fully describe other lesions detected during the operation. In addition, many radiologists are not familiar with CEUS. The radiology community is currently being trained to provide a wide range of professional knowledge and high-level skills.

\section{Conclusion}

The application research of CEUS in other clinical fields has increased exponentially, the capabilities and functions of contrast-enhanced ultrasound are still not fully utilized in practice today. Because the diagnostic performance of CEUS is related to the experience level of the observer. The observer needs to regularly use and understand the relevant physical principles, technical adjustments and the variability of contrast agents. And there is supervision when using UCA for contrast-enhanced ultrasound, But a lot of evidence shows that CEUS can provide unique and accurate diagnostic 
information. CEUS can also be comparable to CT and MRI, and sometimes even better than CT and MRI. In short, CEUS not only has the ability to reduce the difference between diseased and normal tissues in ultrasound imaging, but also has the ability to reduce medical costs, mortality, and ionizing radiation exposure associated with other imaging methods. As more and more evidence reports on the safety and effectiveness of UCA support the use of contrast-enhanced ultrasound and its application in many other medical fields, the advantages of CEUS will be widely understood and accepted by clinicians and researchers. Innovation continues to seek other clinical applications of CEUS to provide safe, effective and valuable diagnosis and treatment options to improve the health of patients.

\section{References}

[1] 王子杨,杨文利.眼科超声检查的发展现状及合理应用 [J].国 际眼科纵览, 2014, 38 (06): 375-379.

[2] Sidhu PS, Cantisani V, Dietrich CF, et al. The EFSUMB Guidelines and Recommendations for the Clinical Practice of Contrast-Enhanced Ultrasound (CEUS) in Non-Hepatic Applications: Update 2017 (Long Version). Die EFSUMB-Leitlinien und Empfehlungen für den klinischen Einsatz des kontrastverstärkten Ultraschalls (CEUS) bei nicht-hepatischen Anwendungen: Update 2017 (Langversion). Ultraschall Med. 2018; 39 (2): e2-e44.

[3] 章希睿, 张明博, 桑茂栋, 等.医学超声造影成像的新技术 研究进展 [J]. 中国生物医学工程学报, 2016, 35 (2): 225-233.

[4] Claudon M, Cosgrove D, Albrecht $\mathrm{T}$ et al. Guidelines and Good ClinicalPractice Recommendations for Contrast Enhanced Ultrasound (CEUS) -Update 2008. Ultraschall in Medizin 2008; 29: 28-44.

[5] Kaufmann BA, Lindner JR. Molecular imaging with target-ed contrast ultrasound. Curr Opin Biotechnol 2007; 18: 11-16 [PMID: 17241779.

[6] Appis, A. W., Tracy, M. J., \& Feinstein, S. B. (2015). Update on the safety and efficacy of commercial ultrasound contrast agents in cardiac applications, Echo Research and Practice, 2 (2), R55-R62.

[7] Nanda NC 1997 History of echocardiographic contrast agents. Clinical Cardiology 20, 7-11.

[8] Feinstein SB, Shah PM, Bing RJ, Meerbaum S, Corday E, Chang BL, Santillan G \& Fujibayashi Y 1984 Microbubble dynamics visualized in the intact capillary circulation. Journal of the American College of Cardiology 4 595-600.

[9] Wei K, Mulvagh SL, Carson L, Davidoff R, Gabriel R, Grimm RA, Wilson S, Fane L, Herzog CA \& Zoghbi WA 2008 The safety of deFinity and Optison for ultrasound image enhancement: a retrospective analysis of 78,383 administered contrast doses. Journal of the American Society of Echocardiography 21 1202-1206.

[10] Wever-Pinzon O, Suma V, Ahuja A, Romero J, Sareen N, Henry SA, De Benedetti Zunino M, Chaudhry FF, SuryadevaraRS \& Sherrid MV 2012 Safety of echocardiographiccontrast in hospitalized patients with pulmonary hypertension: a multi-center study. European Heart Journal Cardiovascular Imaging 13857-862.

[11] 农美芬.超声造影在眼眭肿瘤诊断中的应用[J].微创医学, 2008 (05): 488-490.

[12] 郭佩琦,张吴,王燕霞,马刚, 崔显艳.超声造影联合荧光血管 造影在脉络膜肿瘤诊断中的价值 [J]. 临床超声医学杂志, 2016, 18 (10): 662-666.

[13] Han SS, Chang SK, Yoon JH, Lee YJ. The use of contrast-enhancedcolor Doppler ultrasound in the differentiation of retinal detach-ment from vitreous membrane. Korean J Radiol 2001; 2: 197e203.

[14] Bertolotto, M., Serafini, G., Sconfienza, L. M., Lacelli, F., Cavallaro, M., Coslovich, A., Tognetto, D., \& Cova, M. A. (2014). The use of CEUS in the diagnosis of retinal/choroidal detachment and associated intraocular masses - preliminary investigation in patients with equivocal findings at conventional ultrasound. Ultraschall in der Medizin (Stuttgart, Germany: 1980), 35 (2), 173-180.

[15] 杨文利,魏文斌,李栋军.脉络膜黑色素瘤的超声造影诊断特 征[J].中华眼科杂志, 2013 (05): 428-432.

[16] Piscaglia F, Nolsoe C, Dietrich CF et al. The EFSUMB Guidelines and Re-commendations on the Clinical Practice of Contrast-enhanced Ultra-sound (CEUS): Update 2011 on non-hepatic applications. Ultraschallin Med 2012; 33: 33-59.

[17] Ter Haar G. Safety and bio-effects of ultrasound contrast agents. MedBiol Eng Comput 2009; 47: 893-900.

[18] Migaleddu V, Scanu AM, Quaia E et al. Contrast-Enhanced Ultrasono-graphic Evaluation of Inflammatory Activity in Crohn's Disease. Gas-troenterology 2009; 137: 43-52.

[19] Serra C, Menozzi G, Labate AM, Giangregorio F, Gionchetti P, Beltrami M, Robotti D. et al. Ultrasound assessment of vascularization of the thickened terminal ileum wall in Crohn's disease patients using a low-mechanical index real-time scanning technique with a second generation ultrasound contrast agent. Eur J Radiol 2007; 62: 114-121.

[20] 赵慧, 黄丽萍.超声在炎症性肠病中的应用进展 [J]. 中国医学 影像学杂志, 2020, 28 (09): 717-720.

[21] Strobel D, Seitz K, Blank W, Schuler A, Dietrich C, von Herbay A, Friedrich-Rust M. et al. Contrast-enhanced ultrasound for the characterization of focal liver lesionsdiagnostic accuracy in clinical practice (DEGUM multicenter trial). Ultraschall Med 2008; 29: 499-505.

[22] Strobel D, Bernatik T, Blank W, Schuler A, Greis C, Dietrich CF, Seitz K. Diagnostic accuracy of CEUS in the differential diagnosis of small $(</=20 \mathrm{~mm})$ and subcentimetric $(</=10 \mathrm{~mm})$ focal liver lesions in comparison with histology. Results of the DEGUM multicenter trial. Ultraschall Med 2011; 32: 593-597.

[23] 章建全,陈佳涁,刘灿,蔡清萍,陈学云.经皮经肝穿刺胆道超 声造影的方法及临床意义[J].中华超声影像学杂志, 2008 (06): 513-516.

[24] 谢晓燕,陈瑜君.超声造影的创新性应用 [J].中国医学影像技 术, 2019, 35 (11): 1601-1603.

[25] 李超伦,王文平.超声造影在评价颈动脉粥样硬化斑块稳定 性方面的应用[J].中国临床医学, 2011, 18 (01): 109-111. 
[26] Darge K. Papadopoulu F. Ntoulia A. et al. Safety of contrast-enhanced ultrasound in children for non-cardiac applications: a review by the Society for Pediatric Radiology (SPR) and the International Contrast Ultrasound Society (ICUS). Pediatr Radiol 2013; 43: 1063-1073.

[27] Sidhu PS, Cantisani V, Deganello A, Dietrich CF, Duran C, Franke D, Harkanyi $Z$ et al. Role of Contrast-Enhanced Ultrasound (CEUS) in Paediatric Practice: An EFSUMB Position Statement. Ultraschall Med 2016.
[28] Linde HN. Holland A. Greene BH. et al. Kontrastunterstzte Sonografie (CEUS) bei Pneumonie: Darstellungsmuster und prognostische Bedeutung eine retrospektive Studie bei 50 Patienten. Ultraschall in Med 2012; 33: 146-151.

[29] Durkin N. Deganello A. Sellars ME. et al. Post-traumatic liver and spleen pseudoaneurysms in children: diagnosis, management and follow-up screening using contrast enhanced ultrasound (CEUS). J Pediat Surg 2016; 51: 289-292. 